\title{
ANÁLISE DERIVATIVA DE TESTES DE BOMBEAMENTO EM AQUÍFEROS FISSURAIS NO MUNICÍPIO DE JUNDIAÍ/SP
}

Fábio CRUZ

Elias Hideo TERAMOTO

Juan NAVARRO

Hung Kiang CHANG

\section{RESUMO}

A água subterrânea extraída de aquíferos cristalinos é a principal fonte de água em diversas partes do mundo, particularmente em regiões em desenvolvimento, como África subsaariana, Brasil e Índia. Décadas de pesquisas permitiram constatar uma forte complexidade do regime de fluxo nestes aquíferos, notadamente heterogêneos e anisotrópicos. Tradicionalmente, no Brasil, é assumido que a água se encontra armazenada em descontinuidades geológicas verticalizadas, associadas a zonas pretéritas de falhamentos. Tendo em vista o elevado potencial dos testes de bombeamento para diagnosticar o regime de fluxo, o presente trabalho consistiu na análise derivativa de testes de bombeamento de três poços situados no munícipio de Jundiaí/SP, com vistas ao aprimoramento da compreensão dos modelos conceituais de fluxo em aquíferos fissurais. Tentativas de ajustes com soluções para fraturas verticais e horizontais simples não produziram resultados satisfatórios. Embora nenhuma solução testada tenha se ajustado perfeitamente às curvas de rebaixamento obtidos nos testes de bombeamento, verificou-se que os melhores ajustes foram obtidos com o emprego da solução para aquíferos fraturados com dupla porosidade, compatível com o contexto geológico da área estudada. Novos estudos, similares ao descrito neste trabalho, devem ser conduzidos para o aprimoramento do entendimento das condicionantes de fluxo neste tipo de aquífero.

Palavras-chave: Aquíferos fissurais; Embasamento cristalino; Testes de bombeamento; Análise derivativa; Modelos hidrogeológicos conceituais.

\begin{abstract}
DERIVATIVE ANALYSIS OF PUMPING TESTS CARRIED OUT IN FRACTURED AQUIFERS IN THE JUNDIAÍ MUNICIPALITY - STATE OF SÃO PAULO, BRAZIL. Groundwater extracted from crystalline aquifers is the main water supply in many parts of the world, particularly in developing regions such as Sub-Saharan Africa, Brazil and India. Decades of research have revealed the strong complexity of the flow regime in these aquifers, which are notably heterogeneous and anisotropic. It has been long assumed in Brazil that water is stored in vertical geological discontinuities associated with ancient fault zones. Given the high potential of pumping tests to identify flow regimes, this paper presents a derivative analysis of pumping tests performed in three wells located in the Jundiaí municipality - State of São Paulo, Brazil, aiming to improve the understanding of conceptual flow modeling of fractured aquifers. Adjustment attempts applying solutions for simple vertical and horizontal fractures did not produce satisfactory results. Although no tested solution produced perfect adjustments to the drawdown curves obtained in the pumping tests, the best adjustments were observed when the solution for fractured aquifer with double porosity was applied, which is compatible with the geological context of study area. Further studies, similar to
\end{abstract}


those described in this paper, should be conducted to improve the understanding of flow conditioners for this type of aquifer.

Keywords: Fractured aquifers; Crystalline basement; Pumping tests; Derivative analysis; Conceptual hydrogeological models.

\section{INTRODUÇÃO}

A água representa um recurso vital para a subsistência humana, bem como um fator limitante para o desenvolvimento social e econômico (PAHL-WOSTL et al. 2008). De acordo com SHIKLOMANOV (2000), a exploração de água no mundo saltaria de $331 \mathrm{~km}^{3} /$ ano em 1990 para um valor estimado de $2.182 \mathrm{~km}^{3} /$ ano em 2000 , evidenciando a forte pressão sobre os recursos hídricos, seja ela superficial ou subterrânea. Este fato suscita a necessidade da elaboração de estratégias que visem compreender os processos que governam o fluxo e o armazenamento de água em subsuperfície, para definição de estratégias para a adequada gestão dos recursos hídricos.

Os aquíferos fraturados situados em terrenos cristalinos representam uma importante fonte de abastecimento de água em várias regiões ao redor do mundo, particularmente em regiões subdesenvolvidas (TAYLOR \& HOWARD 2000). Destaca-se que o fluxo e o armazenamento de água em descontinuidades geológicas de rochas cristalinas são notadamente complexos e a capacidade de prever a produtividade desses aquíferos permanece severamente limitada (BERKOWITZ 2002). Como pontuado por diversos trabalhos, destacando-se DEWANDEL et al. (2006), RAFINI \& LAROCQUE (2012), GUIHÉNEUF et al. (2014) e ROQUES et al. (2016), o fluxo de água subterrânea em terrenos cristalinos envolve elevado grau de incertezas. Tais incertezas estão associadas à dificuldade de caracterizar a conectividade e as propriedades hidráulicas das descontinuidades geológicas que armazenam e transmitem água em subsuperfície (GUIHÉNEUF et al. 2014).

A partir de testes hidráulicos em furos de sondagem com 4,5 $\mathrm{km}$ de profundidade, STOBER \& BUCHER (2007) verificaram que os valores de condutividade hidráulica $(\mathrm{K})$ variam 9 ordens de grandeza $\left(10^{-13}-10^{-4} \mathrm{~m} / \mathrm{s}\right)$ em rochas graníticas e gnáissicas, nas quais se verifica um decréscimo exponencial destes valores em função da profundidade. As variações dos valores de $\mathrm{K}$ estão diretamente relacionadas à densidade e à abertura das fraturas em porções mais rasas e sua progressiva diminuição em profundidade (ZHAO 1998, MANNING \& INGEBRITSEN 1999, BOUTT et al. 2010). Por esta razão, intervalos propícios para o armazenamento e circulação de água situam-se em porções mais rasas de subsuperfície (TAYLOR \& HOWARD 2000). A gênese das descontinuidades, passíveis de armazenamento de água, pode ser atribuída à diversos fatores distintos, relacionados a eventos tectônicos, intempéricos e de descompressão.

Os esforços tectônicos ao longo de falhas transcorrentes são responsáveis pela gênese de uma zona intensamente fraturada, normalmente limitada à profundidade de poucas centenas de metros (GUDMUNDSSON 1992, CAINE et al, 1996, BENSE et al. 2013). De acordo com CAINE et al. (1996), os falhamentos são responsáveis pela gênese de um zoneamento com valores distintos de permeabilidade, a saber: centro da falha, zona de dano e protólito. O centro da falha, composto por milonitos e cataclasitos, e o protólito possuem, ambos, valores bastante reduzidos de permeabilidade. Em oposição, a zona de dano, representada por regiões intensamente fraturadas e desenvolvidas em torno das zonas de falhas, possui valores elevados de condutividade hidráulica (CAINE et al. 1996). A viabilidade deste modelo conceitual foi comprovada por inúmeros autores, dentre os quais, MABEE et al. (1994), NEVES \& MORALES (2007), FAULKNER et al. (2010), RAFINI \& LAROCQUE (2012), ROQUES et al. (2016), cujos resultados demonstraram que zonas com elevados valores de transmissividade situam-se próximos a zonas de falhas.

Diversos trabalhos têm demonstrado que o faturamento de rochas cristalinas pode ser decorrente de processos intempéricos (DEWANDEL et al. 2006, LACHASSAGNE et al. 2011). NETTLETON et al. (1970) verificaram que a transformação da biotita em vermiculita durante o processo intempérico é responsável por incrementos de $40 \%$ no volume da rocha. Por esta razão, LACHASSAGNE et al. (2011) assumem que o intemperismo da biotita, importante mineral de rochas cristalinas, promove um incremento no volume do maciço, criando um stress, que é responsável pelo fraturamento da rocha.

Outros trabalhos, tais como ACWORTH (1987), WRIGHT (1992), TAYLOR \& HOWARD (2000) e HOLBROOK et al. (2019), assumem que o principal agente gerador do horizonte fissural é o alívio por descompressão litostástica (unload). De 
acordo com este modelo, a erosão de parte do maciço, a partir da evolução geomórfica do terreno, gera uma dilatação vertical do maciço rochoso à medida que a pressão litostástica é reduzida, promovendo a geração de fraturas ao longo de linhas de fraqueza próximas à superfície. Essas fraturas, chamadas de juntas, geralmente são paralelas ou subparalelas à superfície (TAYLOR \& HOWARD 2000).

A despeito dos mecanismos responsáveis pela gênese das fraturas em terrenos cristalinos, a distribuição destas estruturas é notadamente complexa, conferindo propriedades hidrodinâmicas invariavelmente heterogêneas e anisotrópicas. Em razão desta complexidade, testes de bombeamento representam elementos cruciais para a determinação dos parâmetros hidráulicos deste tipo de aquífero, como demonstrado por inúmeros trabalhos, como os de WRIGHT (1992), TAYLOR \& HOWARD (2000), LE BORGNE et al. (2004), MARÉCHAL et al. (2004) e VOUILLAMOZ et al. (2005).

Os fatores condicionantes na produtividade dos poços tubulares profundos que extraem água de aquíferos cristalinos na região de Jundiaí/SP foram previamente estudados por NEVES \& MORALES (2007). A despeito das importantes contribuições dadas por este trabalho, vários aspectos não foram devidamente elucidados, em razão da complexidade e da diversidade de mecanismos responsáveis pela geração de descontinuidades geológicas passíveis de armazenar água em terrenos cristalinos. Para complementar o entendimento do armazenamento e a circulação de água em terrenos cristalinos na região de Jundiaí/SP, foi objetivo deste trabalho empregar análise de testes de bombeamento, com o intuito de se identificar os modelos conceituais mais apropriados para a dinâmica de água subterrânea na área de interesse.

\section{GEOLOGIA REGIONAL}

A Bacia do Rio Jundiaí é marcada por forte complexidade geológica, onde afloram rochas metassedimentares pré-cambrianas do Grupo São Roque, granitoides sin- e pós-tectônicos, rochas sedimentares do Subgrupo Itararé, depósitos terciários correlatos à Formação Rio Claro e depósitos aluvionares quaternários (Figura 1).

Das unidades aflorantes na área de estudo, merece destaque o Complexo Amparo, que é a unidade de maior representatividade. Esta unidade é predominantemente composta por gnaisses com biotita, hornblenda e granada, com grau variável de migmatização e intercalações de quartzitos, xistos, anfibolitos, gonditos e metaultrabasitos (HASUI et al. 1981).

A estrutura geológica mais proeminente na Bacia do Rio Jundiaí é a Zona de Cisalhamento Jundiuvira, que se ramifica na porção oeste da ba-

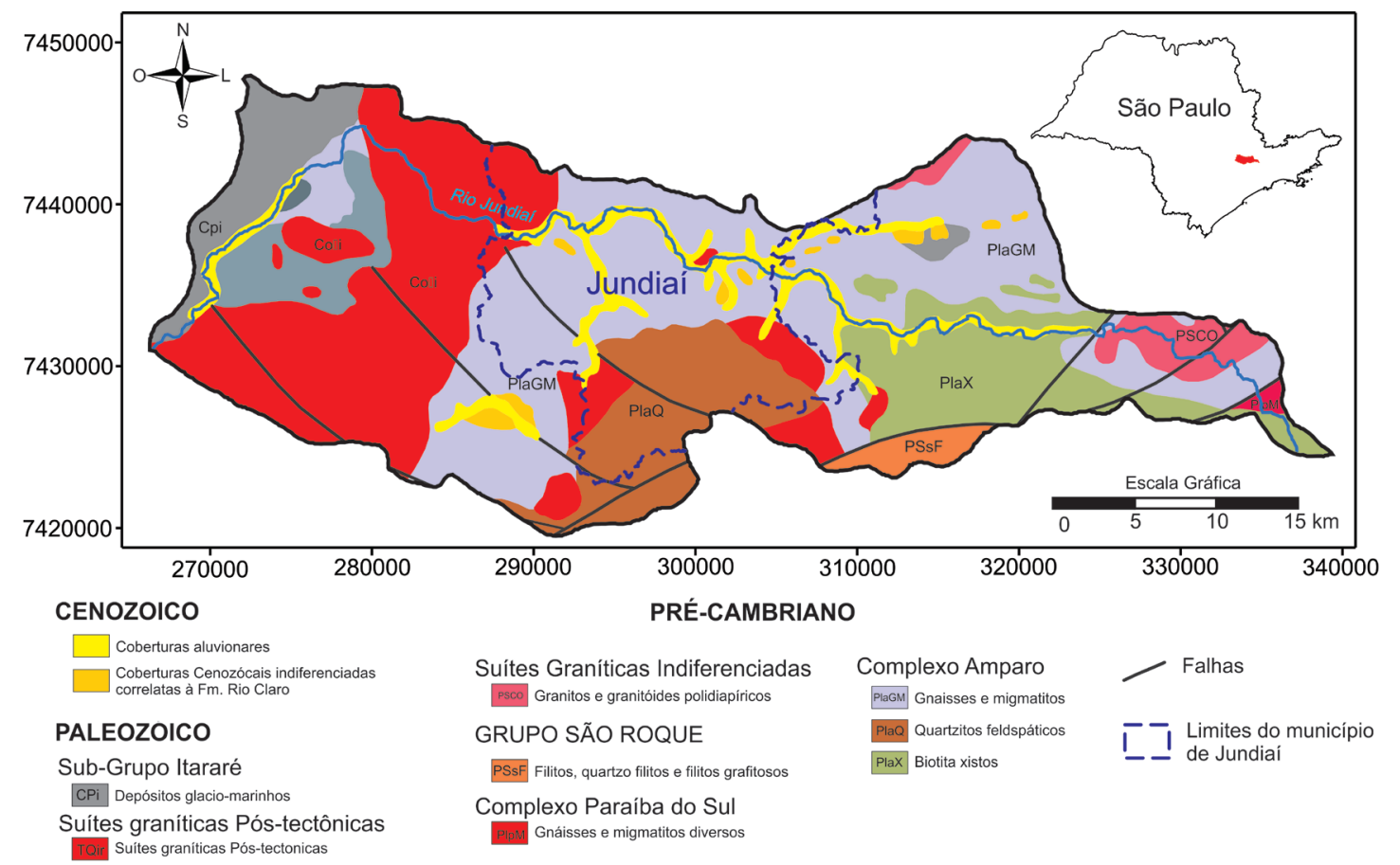

FIGURA 1 - Mapa geológico simplificado da Bacia do Rio Jundiaí (adaptado de IRRIGART 2005). 
cia nas zonas de cisalhamento de Itu, Piraí, Cururu e Cachoeira em feixes de direção NW-SE (HASUI et al. 1978), em uma estrutura rabo de cavalo. De acordo com HASUI et al. (1978), a Zona de Falha de Jundiuvira está associada à malha de grandes falhas transcorrentes e zonas de cisalhamento que controlaram a estruturação do embasamento cristalino, apresentando movimentação geral transcorrente destral.

\section{MATERIAIS E MÉTODOS}

\section{1 Área de estudo}

Os três poços de abastecimento onde os ensaios de rebaixamento foram realizados estão situados na porção centro-oeste do munícipio de Jundiaí/ SP (Figura 2), a $60 \mathrm{~km}$ da capital paulista. Nessa região afloram rochas gnáissicas e migmatíticas do Complexo Amparo. O município de Jundiaí está inserido dentro da Bacia do Rio Jundiaí, que ocupa uma área de 1.114,03 km² (IRRIGART 2005).

3.1 Testes de bombeamento realizados em aquíferos fissurais

Os três poços analisados foram instalados em aquíferos fissurais compostos por gnaisses migmatíticos do Complexo Amparo. A figura 3 ilustra o perfil geológico e construtivo simplificado dos três poços analisados.

Os testes de bombeamento consistiram na extração ininterrupta de um volume constante de água por um período de $24 \mathrm{~h}$, com medição da vazão e rebaixamento ao longo do tempo. O rebaixamento foi medido no próprio poço bombeado, utilizando-se um medidor elétrico graduado de nível d'água. A vazão do poço foi periodicamente mensurada com auxílio de um recipiente graduado e cronômetro, de modo a avaliar as variações de vazão ao longo do teste. Após o período de $24 \mathrm{~h}$ de teste, o bombeamento foi interrompido e a recuperação do nível nos poços foi igualmente monitorada até o nível d'água retornar ao seu nível estático original.

\subsection{Curvas derivativas}

O trabalho BOURDET et al. (1989) propôs uma metodologia na qual, além de plotar os dados de pressão (p), eram também plotados os valores da derivada da pressão $\mathrm{dp} / \mathrm{d}(\log (\mathrm{t}))$ para diagnósticos mais precisos do regime de fluxo em reservatórios de petróleo. RENARD et al. (2009) demonstraram que esta abordagem era igualmente funcional para diagnosticar o tipo de fluxo de água subterrânea, a partir da derivada de ds/dlog(t) (derivada do rebaixamento em função do tempo em escala logaritimica) $(\mathrm{L} / \mathrm{T})$, tendo em vista que a derivativa do rebaixamento (s) é muito sensível a pequenas variações na taxa de rebaixamento.

A representação gráfica da curva diagnóstica, combinação de s (L) e ds/dlog(t) versus tempo, é utilizada para melhorar a interpretação dos testes de bombeamento. O cálculo da curva derivativa de testes de bombeamento é realizado empregando a me-

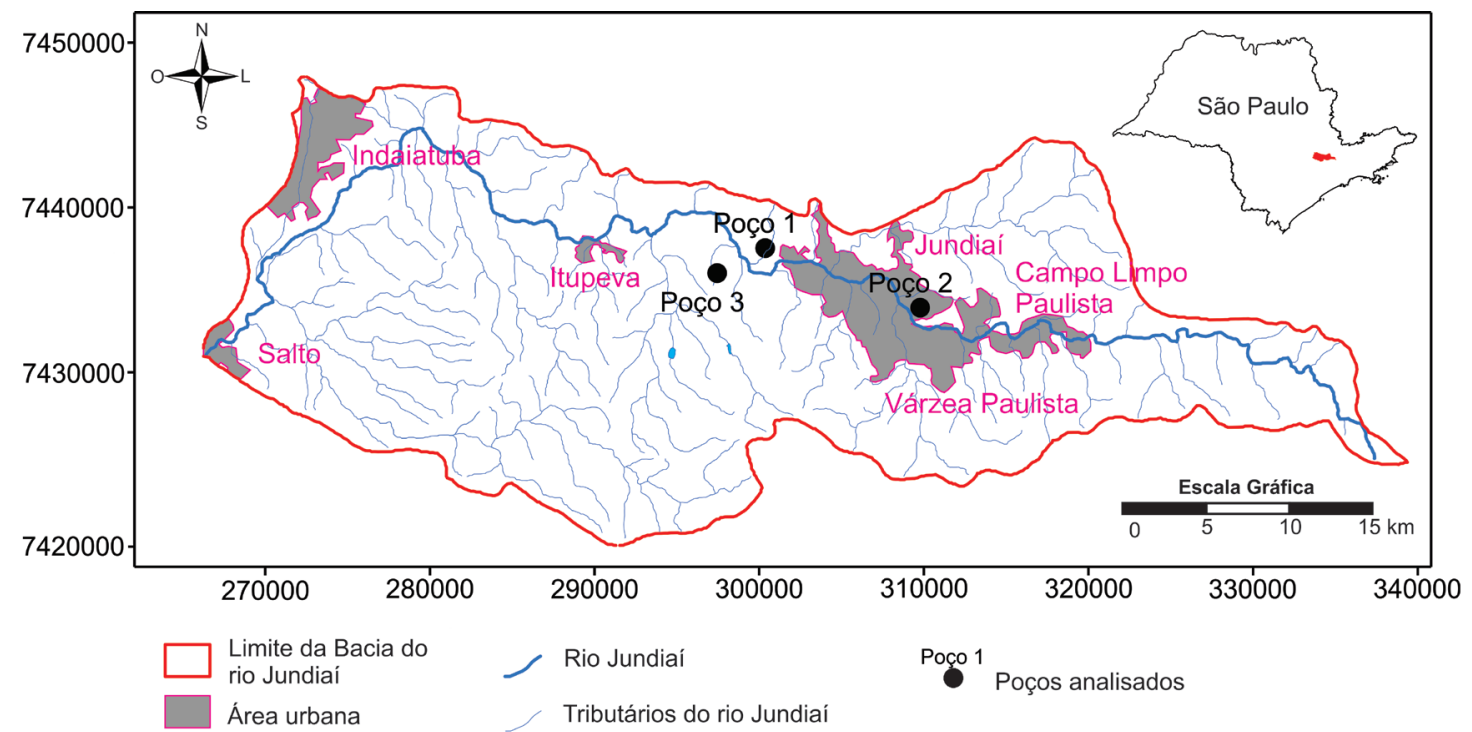

FIGURA 2 - Localização dos poços onde foram executados os testes de bombeamento interpretados no presente trabalho. A área de estudo situa-se no munícipio de Jundiaí, inserida na bacia do rio homônimo. 


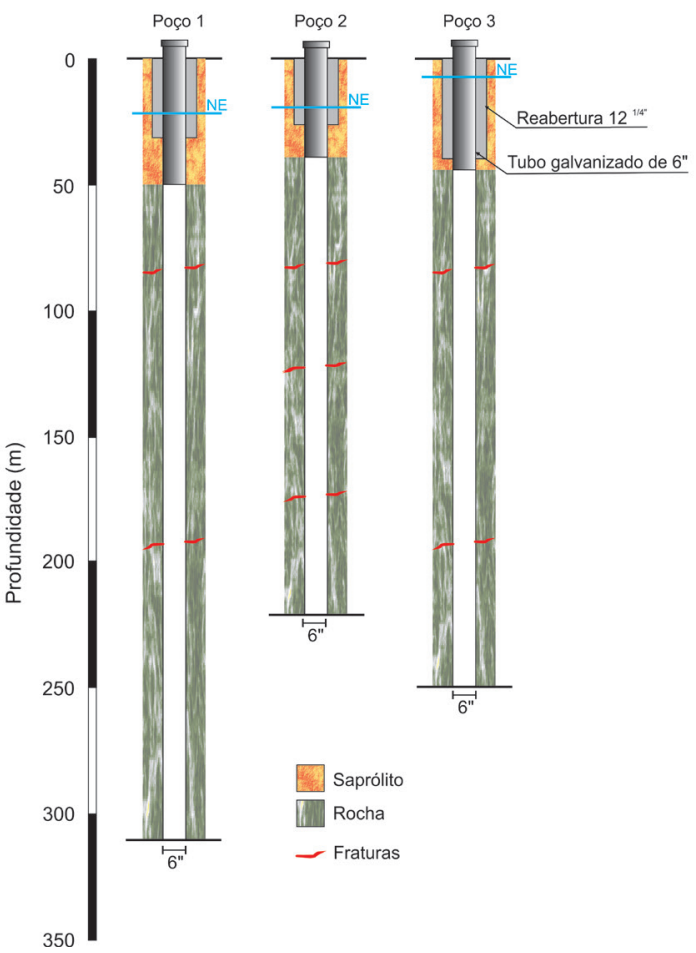

FIGURA 3 - Perfis construtivos e geológicos simplificados dos três poços tubulares avaliados, instalados em rochas fissurais do Complexo Amparo.

todologia de BOURDET et al. (1989), que aproxima a derivada da curva de rebaixamento por meio da equação 1 .

$$
\left(\frac{\partial s}{\partial \ln t}\right)=\frac{\left(\frac{\partial s_{i-1}}{\partial \ln t_{i-1}}\right) \Delta \ln t_{i+1}+\left(\frac{\partial s_{i+1}}{\partial \ln t_{i+1}}\right) \Delta \ln t_{i-1}}{\Delta \ln t_{i-1}+\Delta \ln t_{i+1}}
$$

BARKER (1988) introduziu o conceito de dimensão do fluxo $(\mathrm{n})$, relacionada à área $\mathrm{A}\left(\mathrm{L}^{2}\right)$, a partir da qual o fluxo ocorre (Equação 2).

$$
A(r) \sim r^{(n-1)}
$$

onde r é a distância entre o poço de bombeamento e a frente de pulso. $\mathrm{O}$ valor de $\mathrm{n}$ traduz o tipo de fluxo no interior do aquífero durante certo estágio do teste de bombeamento, possuindo valor igual a 1 para fluxos lineares, 1,5 para fluxos bilineares, 2 para fluxos radiais e 3 para fluxos esféricos. A construção da curva derivativa, empregando a equação 1 , permite avaliar variações de $\mathrm{n}$ ao longo do teste (Figura 4), como demonstrado por EHLIG-ECONOMIDES et al. (1994). O

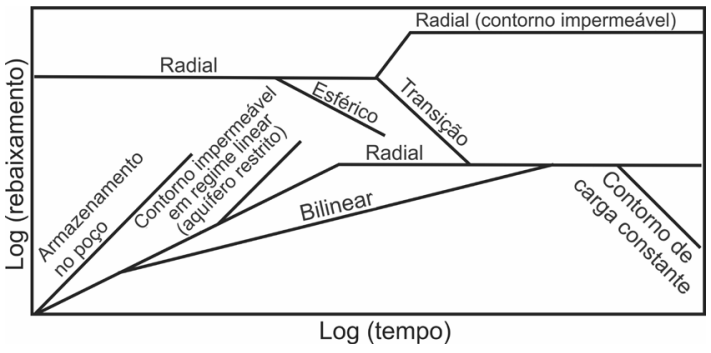

FIGURA 4 - Diagrama para identificação de regime de fluxo que representa esquematicamente a derivada logarítmica do rebaixamento em função do tempo em escala logarítmica (Modificado de EHLIGECONOMIDES et al. 1994).

diagrama pode ser sobreposto aos dados e alterados para identificar visualmente as dimensões de fluxo para construção das curvas diagnósticas e identificação dos regimes de fluxos.

Como ilustrado na figura 4, o trecho inicial da curva derivativa pode indicar armazenamento no interior do poço, a porção média reflete a natureza de aquífero, enquanto sua parte final, a presença e o tipo de condições de contorno. Por esta razão, a sucessão de diferentes dimensões de fluxo gera uma curva diagnóstica, que permite discernir sobre o regime de fluxo que melhor se ajusta ao rebaixamento em função do tempo, como demonstrado por RENARD et al. (2009). O método mais usual para interpretar a curva derivativa consiste na tentativa de ajustes de uma curva teórica àquela obtida a partir dos dados observados. A figura 5 ilustra quatro exemplos de curvas de rebaixamento e suas respectivas curvas diagnósticas para contextos hidrogeológicos distintos.

\subsection{Interpretação dos testes de bombeamento}

Para a interpretação dos testes de bombeamento, utilizou-se os dados construtivos (profundidade e diâmetro do poço), de rebaixamento e de vazão dos três poços avaliados no presente trabalho. Os dados foram inseridos no aplicativo AQTESOLV $^{\circledR}$ para a geração das curvas de rebaixamento e de derivada do rebaixamento para cada um dos poços.

A partir das curvas derivativas construídas, buscou-se identificar o modelo de aquíferoque melhor reproduz as curvas de rebaixamento obtidas. Uma vez que cada tipo de aquífero possui uma curva derivativa diagnóstica (exemplos ilustrados na figura 5), foi possível identificar a 


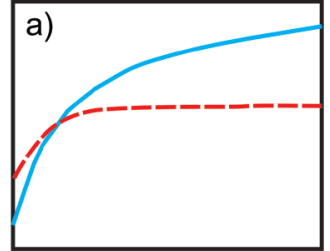

$\log (\mathrm{t})$

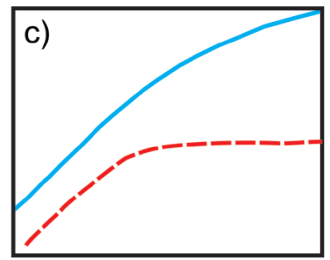

$\log (\mathrm{t})$

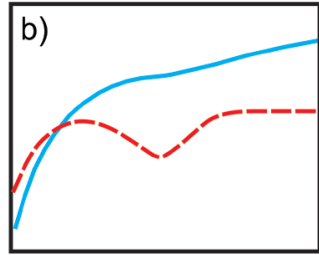

$\log (\mathrm{t})$

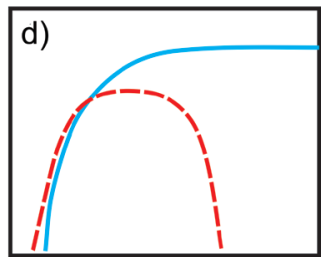

$\log (\mathrm{t})$
Curva de rebaixamento

Curva derivativa

FIGURA 5 - Exemplos de algumas curvas diagnósticas: a) Confinado; b) dupla porosidade ou aquífero livre; c) fratura vertical simples; c) confinado drenante (Adaptado de RENARD et al. 2009).

solução mais apropriado para reproduzir a curva de rebaixamento obtida no teste. É necessário destacar que diferentes modelos de aquíferos podem possuir curvas derivativas similares, tais como fraturado com dupla porosidade e aquífero livre (Figura 5a); desse modo, optou-se por aquela compatível com o contexto hidrogeológico em análise.

Após a seleção da solução mais apropriada, foi realizado o ajuste das curvas do modelo às curvas de rebaixamento obtidas nos três poços testados, conduzido a partir de adequações sucessivas nos diversos parâmetros hidrodinâmicos que servem como parâmetros de entrada. Uma vez obtido o melhor ajuste com a solução selecionada, foram anotados os parâmetros hidrodinâmicos (condutividade hidráulica da matriz ou fratura, armazenamento, dimensão de fluxo, etc.) correspondentes.

\section{RESULTADOS}

\subsection{Testes de bombeamento}

A figura 6 ilustra as variações de vazão ao longo dos três testes de bombeamento efetuados na área de estudo. É possível notar uma queda exponencial da vazão na parte inicial do teste e posterior estabilização da vazão. Embora tenha-se

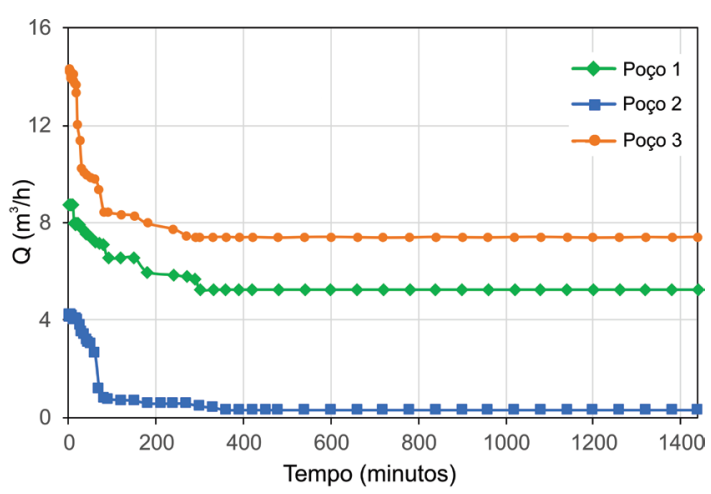

FIGURA 6 - Variações de vazão durante a execução dos testes de bombeamentos para os três ensaios analisados.

tentado manter a vazão máxima constante, foram registradas pequenas oscilações na vazão do bombeamento, as quais podem ser decorrentes de variações na velocidade de rotação da bomba, oscilação da corrente elétrica ou efeitos da geometria do aquífero avaliado.

\subsection{Ajustes dos testes}

Para identificar o modelo hidrogeológico mais representativo do regime local, foram testados os modelos de GRINGARTEN \& WITHERSPOON (1972) para fraturas verticais simples, GRINGARTEN \& RAMEY (1974) para fraturas horizontais simples, BARKER (1988) para aquíferos fraturados com dupla porosidade, MOENCH (1997) para aquíferos porosos livres.

Nos poços 1, 2 e 3 as soluções de GRINGARTEN \& WITHERSPOON (1972) (Figura 7) e GRINGATEN \& RAMEY (1974) (Figura 8) forneceram péssimos ajustes, enquanto as soluções de BARKER (1988) (Figura 9), e MOENCH (1997) (Figura 10), forneceram bons ajustes, com qualidade similar. Entretanto, assume-se que o primeiro ofereça o resultado mais plausível, uma vez que as soluções para aquíferos livres (MOENCH 1997) não se justifica no contexto hidrogeológico estudado. Nota-se que os ajustes foram piores na porção inicial da curva, refletindo a incapacidade da solução de BARKER (1988) representar o fluxo da água armazenada no poço.

Os parâmetros hidrodinâmicos obtidos para o ajuste do modelo de BARKER (1988) dos três poços analisados (Figura 9) são apresentados na tabela 1 . 

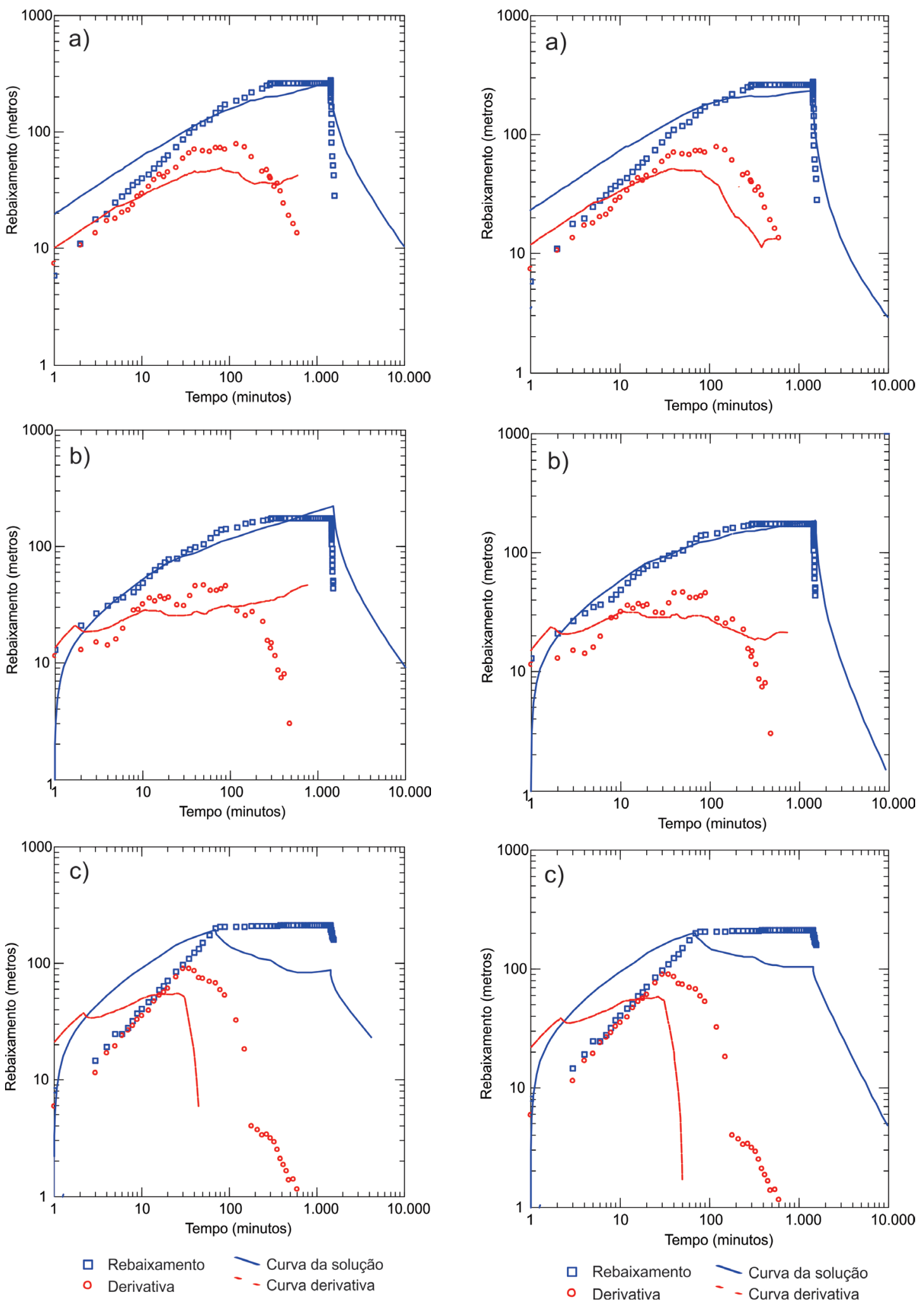

FIGURA 7-Ajustes com a solução de GRINGARTEN \& WHITERSPOON (1972) para aquíferos fraturados com fratura vertical simples: a) Poço 1; b) Poço 2; c) Poço 3.

FIGURA 8 - Ajustes com a solução de GRINGARTEN \& RAMEY (1974) para aquíferos fraturados com fratura horizontal simples: a) Poço 1; b) Poço 2; c) Poço 3. 

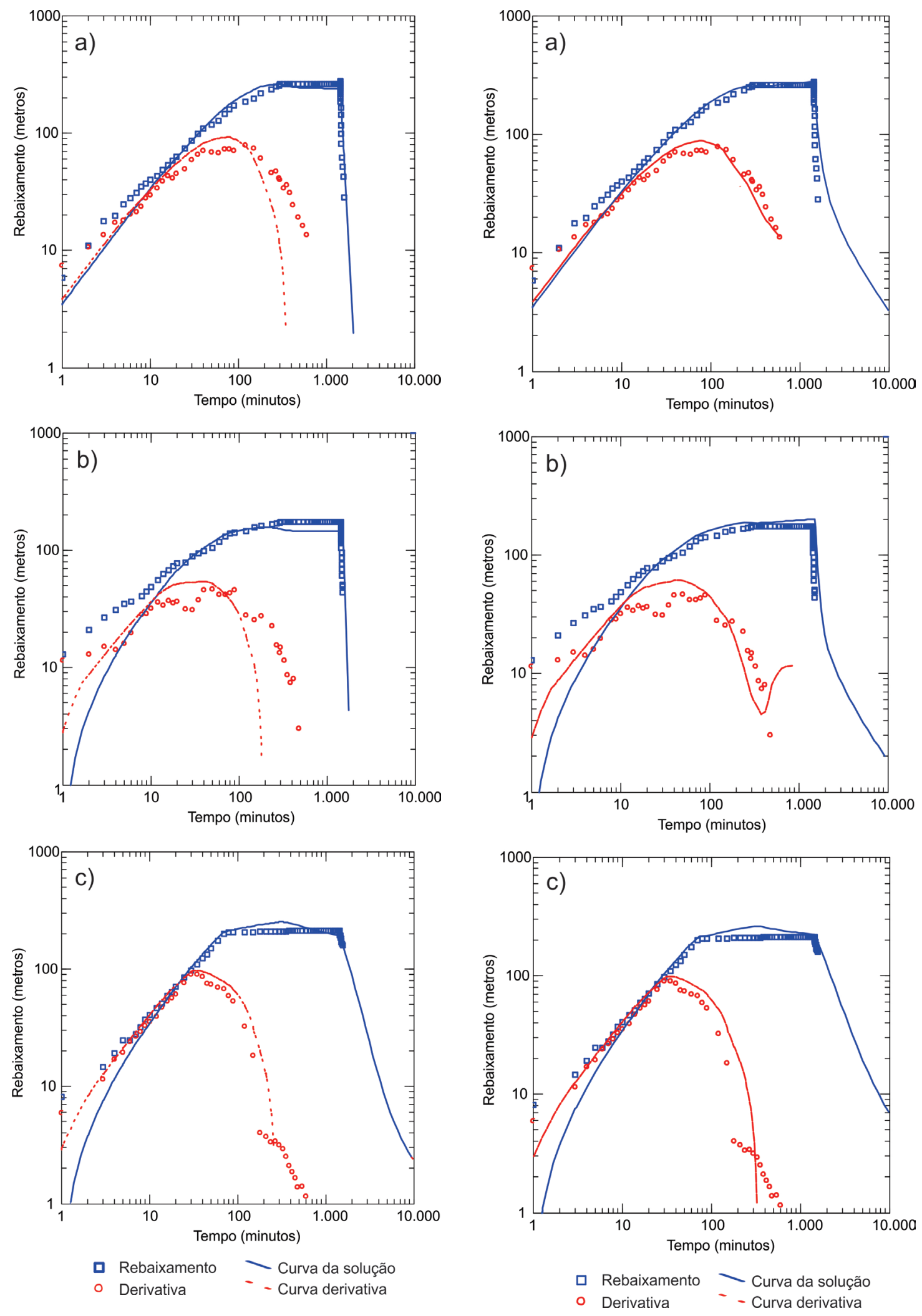

FIGURA 9 - Ajustes com a solução de BARKER (1988) para fraturados com dupla porosidade: a) Poço 1; b) Poço 2; c) Poço 3.

FIGURA 10 - Ajustes com a solução de MOENCH (1997) para aquíferos porosos livres: a) Poço 1; b) Poço 2; c) Poço 3. 
TABELA 1 - Parâmetros obtidos pelo ajuste do modelo de BARKER (1988) para as três curvas de rebaixamento analisadas.

\begin{tabular}{crrr}
\hline Parâmetro & \multicolumn{1}{c}{ Poço 1 } & \multicolumn{1}{c}{ Poço 2 } & \multicolumn{1}{c}{ Poço 3 } \\
\hline K - fratura (m/dia) & 0,7049 & 0,4637 & 1,453 \\
Ss - fratura & $1,90 \times 10^{-5}$ & $2,59 \times 10^{-5}$ & $2,07 \times 10^{-5}$ \\
K - matriz (m/dia) & $7,22 \times 10^{-6}$ & $2,28 \times 10^{-7}$ & $8,09 \times 10^{-7}$ \\
Ss - matriz & $1,0 \times 10^{-5}$ & $1,0 \times 10^{-5}$ & $2,5 \times 10^{-5}$ \\
b & 834,4 & 436,5 & 289,3 \\
Sf & 7,75 & 780 & 83 \\
Sw & 0,825 & 10 & 0,82 \\
\hline
\end{tabular}

$\mathrm{K}=$ condutividade hidráulica, $\mathrm{Ss}=$ Armazenamento, $\mathrm{n}=$ dimensão de fluxo da fratura, $\mathrm{b}=$ extensão da região de fluxo, $\mathrm{Sf}=$ perda de carga devido às características da parede do poço, $\mathrm{S}_{\mathrm{w}}=$ capacidade de armazenamento na fonte.

\section{DISCUSSÃO}

As diversas soluções analíticas elaboradas para descrever o fluxo em poços que extraem água subterrânea congregam uma série de premissas válidas para condições hidrogeológicas específicas. De acordo com RENARD et al. (2009) e FERROUD et al. (2018), realizar ajustes bem-sucedidos de soluções analíticas específicas permite assumir que as suas premissas são extensíveis aos aquíferos onde os testes de bombeamento foram executados. A discrepância entre a complexidade do comportamento do fluxo real e a simplicidade dos modelos de fluxo analítico tornam a interpretação dos testes de bombeamento uma tarefa ambígua e imprecisa, quando as técnicas derivativas não são utilizadas.

As soluções analíticas de THEIS (1935) e COOPER \& JACOB (1946) representam as soluções analíticas mais comumente empregadas para interpretação dos testes de bombeamento. Contudo, tais soluções são excessivamente simplistas, assumindo a premissa de um regime de fluxo do tipo radial infinito, que pode ser ajustado graficamente por uma reta. Tendo em vista que o fluxo radial está presente em um trecho limitado da curva de rebaixamento em aquíferos complexos, as soluções de THEIS (1935) e COOPER \& JACOB (1946) não são apropriadas para a sua interpretação. Por esta razão, as propriedades hidráulicas obtidas a partir de soluções inadequadas podem levar a aproximações grosseiras e estimativas equivocadas, como demonstrado por FERROUD et al. (2018).
Além da complexidade intrínseca a diversos contextos hidrogeológicos distintos, é importante destacar que a interpretação de testes de bombeamento pode guardar ambiguidades, uma vez que, frequentemente, mais de uma solução pode ser ajustada a esta curva de rebaixamento. Parcela significativa desta ambiguidade pode ser eliminada a partir da análise de curvas derivativas, que permitem diagnosticar o modelo conceitual mais adequado à curva de rebaixamento, embora o emprego desta técnica seja pouco comum no Brasil.

Baseando-se na premissa de que a água está armazenada em descontinuidades geológicas associadas às zonas de falhas, a prospecção de água em terrenos tem sido tradicionalmente conduzida por mapeamento de alinhamentos estruturais (MABEE et al. 1994, BABIKER \& GUDIMUNSSON 2004, GALANOS \& ROKOS 2006, NEVES \& MORALES 2007, BRUNING et al. 2011, BHUIYAN 2015, MADRUCCI 2003, AYER et al. 2017). Tal abordagem pressupõe que a água está, na quase totalidade dos casos, armazenada em zonas de falhas. Embora tais premissas possam ser verdadeiras em diversos casos, elas são incapazes de explicar a existência de poços produtivos distantes de alinhamentos estruturais reconhecidos regionalmente, caso dos poços analisados no presente estudo. Se a água subterrânea na área de estudo estiver armazenada em descontinuidades verticais, é esperado que a curva de rebaixamento seja ajustada pela solução de GRINGARTEN \& WITHERSPPON (1972), que descreve a solução para um modelo com fratura vertical simples. No entanto, como é possível observar na Figura 7, não foi possível ajustar satisfatoriamente a curva de rebaixamento com esta solução, sugerindo que a água não está armazenada em descontinuidades verticais.

Tentativas de ajustes com as diversas soluções testadas indicam que as três curvas de rebaixamento podem ser ajustadas com a mesma qualidade de ajuste empregando os modelos de MOENCH (1997) para aquíferos porosos livres, e BARKER (1988) para aquíferos fraturados com dupla porosidade. Nestes casos, onde mais de uma solução se ajusta aos testes avaliados, o contexto geológico deve ser detalhadamente investigado para selecionar o modelo mais apropriado.

De acordo com as premissas apresentadas por DEWANDEL et al. (2006) e LACHASSAGNE et al. (2011), os processos intempéricos são responsáveis pela gênese de 
fraturas horizontalizadas. Se tal constatação fosse verdadeira para a área de estudo, seria esperado um bom ajuste com o modelo de GRINGARTEN \& RAMEY (1974), o que não foi observado nos três testes de bombeamento.

LEE \& LEE (2000), a partir de ensaios de traçadores, verificaram que meios fraturados podem se assemelhar a um meio poroso, quando a densidade de fraturas é elevada e estas estão orientadas randomicamente. Obteve-se um bom ajuste empregando a solução para aquífero poroso livre de MOENCH (1997), como observado na Figura 10, similarmente ao observado no trabalho de PERSAUD et al. (2018). No entanto, os perfis geológicos dos poços analisados não indicam a existência de intervalos intensamente fraturados.

O avanço do intemperismo é responsável por significativo incremento na matriz de rochas cristalinas (TAYLOR \& HOWARD 2000, HOLBROOK et al. 2019). É provável que este incremento de porosidade gere um reservatório com dupla porosidade, na qual coexistam dois grupos de poros distintos, o primeiro representado pelas fraturas e o segundo representado pela porosidade da matriz da rocha. REIMUS et al. (2003) empregando ensaios com múltiplos traçadores verificaram que o modelo de dupla porosidade no Estado de Nevada, Estados Unidos, é o modelo mais apropriado para explicar o transporte de solutos em rochas cristalinas fraturadas. Este fato é corroborado pelos testes de bombeamentos interpretados por MARÉCHAL et al. (2004), que verificaram um regime de fluxo com dupla porosidade, formado pela rede de fraturadas e pela matriz, em terrenos graníticos da Índia. Diante do contexto geológico observado na região de Jundiaí (SP) e descrito nos perfis geológicos durante a perfuração dos poços, assume-se que a solução de BARKER (1988), para aquíferos fraturados com dupla porosidade, represente a solução mais adequada. Alternativamente, como demonstrado por JOURDE et al. (2002), a presença de dois conjuntos de fraturas, com orientações e valores distintos de condutividade hidráulica, pode gerar curvas derivativas de dupla porosidade. A curva derivativa observada nos três poços analisados difere daquela esperada para aquíferos com dupla porosidade, com geometria em "V" (Figura 5b), fato que pode ser atribuído ao tempo de duração do teste $(24 \mathrm{~h})$, impedindo que todas as dimensões de fluxo fossem reproduzidas na curva obtida.
Os resultados sugerem que a água no aquífero cristalino do munícipio de Jundiaí não está armazenada em fraturas simples, sejam elas verticais ou horizontais. Este resultado se opõe a trabalhos anteriores desenvolvidos na área de estudo e indicam que a água se encontra armazenada em intervalos fraturados onde a contribuição da matriz é relevante. Embora o universo amostral seja bastante reduzido, os resultados indicam que o regime de fluxo em meios descontínuos na área de estudo é mais complexo do que o previsto a prio$r i$, na qual se assume que a água esteja armazenada em fraturas verticais geradas por esforços tectônicos distensivos. Por esta razão, a metodologia tradicionalmente empregada no Brasil, baseada na identificação de lineamentos por fotografias aéreas ou sensoriamento remoto, podem ser pouco úteis, além de simplistas. As constatações obtidas no presente trabalho não se restringem apenas à área de estudo, podendo se estender a outros aquíferos cristalinos fraturados. Há necessidade de se reavaliar os modelos conceituais vigentes no Brasil para prospecção e gestão de recursos hídricos subterrâneos em aquíferos cristalinos.

No Brasil, a interpretação dos testes de bombeamento restringe-se ao simples ajuste das soluções de Theis e Cooper-Jacob nas curvas de rebaixamento para obtenção dos valores de transmissividade e armazenamento. Observou-se neste trabalho a potencialidade das análises derivativas das curvas de rebaixamento para diagnósticos mais precisos dos regimes de fluxo dos aquíferos testados, permitindo assim, um entendimento mais acurado do aquífero analisado.

\section{CONCLUSÕES}

Para uma investigação preliminar do regime de fluxo em aquíferos cristalinos na região de Jundiaí/SP, três testes de bombeamento foram detalhadamente analisados. Em razão do contexto geológico verificado na área de estudo, era esperado que o regime de fluxo fosse ajustado por soluções que descrevem o fluxo em fraturas simples, sejam elas verticais ou horizontais. No entanto, os três testes de bombeamento avaliados no presente trabalho sugerem que o regime de fluxo em rochas fissuradas da região de Jundiaí/ SP podem ser ajustados por soluções para aquíferos fraturados com dupla porosidade. Neste tipo de solução, assume-se que a água que flui para o poço bombeado é proveniente do armazenamento nas fraturas e na matriz da rocha. 


\section{AGRADECIMENTOS}

Os autores agradecem à empresa UNISONDAS pela cessão dos dados construtivos e dos testes de bombeamento dos poços utilizados no presente trabalho, a FUNDUNESP pela concessão de bolsas de pesquisa e ao LEBAC pela infraestrutura e aplicativos computacionais necessários para o desenvolvimento do presente trabalho. Aos relatores da Revista do Instituto Geológico pelas sugestões apresentadas, que enriqueceram o trabalho.

\section{REFERÊNCIAS BIBLIOGRÁFICAS}

ACWORTH, R.I. 1987. The development of crystalline basement aquifers in a tropical environment. Quarterly Journal of Engineering Geology and Hydrogeology, 20(4): 265-272. https://doi.org/10.1144/GSL. QJEG.1987.020.04.02

AYER, J.E.B.; GAROFALO, D.F.T.; PEREIRA, S.Y. 2017. Uso de geotecnologias na avaliação da favorabilidade hidrogeológica em aquíferos fraturados. Águas Subterrâneas, 31(3): 154-167. https://dx.doi.org/10.14295/ ras.v31i3.28773

BABIKER, M.; GUDMUNDSSON, A. 2004. The effects of dykes and faults on groundwater flow in an arid land: the Red Sea Hills, Sudan. Journal of Hydrology, 297(14): 256-273. https://doi.org/10.1016/j. jhydrol.2004.04.018

BARKER, J.A. 1988. A generalized radial flow model for hydraulic tests in fractured rock. Water Resources Research, 24(10): 1796-1804. https://doi.org/10.1029/ WR024i010p01796

BENSE, V.F.; GLEESON, T.; LOVELESS, S.E.; BOUR, O.; SCIBEK, J. 2013. Fault zone hydrogeology. Earth-Science Reviews, 127: 171-192. https://doi.org/10.1016/j. earscirev.2013.09.008

BERKOWITZ, B. 2002. Characterizing flow and transport in fractured geological media: A review. Advances in Water Resources, 25(8-12): 861-884. https://doi.org/10.1016/ S0309-1708(02)00042-8
BHUIYAN, C. 2015. Hydrological characterisation of geological lineaments: a case study from the Aravalli terrain, India. Hydrogeology Journal, 23(4): 673-686. https://doi. org/10.1007/s10040-015-1239-0

BOURDET, D.; AYOUB, J.A.; PIRARD, Y.M. 1989. Use of pressure derivative in well test interpretation. SPE Formation Evaluation, 4(02): 293-302. https://doi. org/10.2118/12777-PA

BOUTT, D.F.; DIGGINS, P.; MABEE, S. 2010. A field study (Massachusetts, USA) of the factors controlling the depth of groundwater flow systems in crystalline fractured-rock terrain. Hydrogeology Journal, 18(8): 18391854. https://doi.org/10.1007/s10040-0100640-y

BRUNING, J.N.; GIERKE, J.S.; MACLEAN, A.L. 2011. An approach to lineament analysis for groundwater exploration in Nicaragua. Photogrammetric Engineering Remote Sensing, 77(5): 509-519. https://doi. org/10.14358/PERS.77.5.509

CAINE, J.S., EVANS, J.P., FORSTER, C.B. 1996. Fault zone architecture and permeability structure. Geology, 24(11): 1025-1028.https:// doi.org/10.1130/0091-7613(1996)024\%3C10 25:FZAAPS\%3E2.3.CO;2

COOPER Jr., H.H.; JACOB, C.E. 1946. A generalized graphical method for evaluating formation constants and summarizing wellfield history. Eos, Transactions American Geophysical Union, 27 (4): 526-534. https:// doi.org/10.1029/TR027i004p00526

DEWANDEL, B.; LACHASSAGNE, P.; WYNS, R.;MARÉCHAL, J.C.; KRISHNAMURTHY, N.S. 2006. A generalized 3-D geological and hydrogeological conceptual model of granite aquifers controlled by single or multiphase weathering. Journal of Hydrology, 330(12): 260-284. https://doi.org/10.1016/j. jhydrol.2006.03.026

EHLIG-ECONOMIDES, C.A.; HEGEMAN, P.; VIK, S. 1994. Guidelines simplify well test interpretation. Oil \& Gas Journal, 92(29), $33 \mathrm{p}$. 
FAULKNER, D.R., JACKSON, C.A.L.; LUNN, R.J.; SCHLISCHE, R.W.; SHIPTON, Z.K.; WIBBERLEY, C.A.J.; WITHJACK, M.O. 2010. A review of recent developments concerning the structure, mechanics and fluid flow properties of fault zones. Journal of Structural Geology, 32(11): 1557-1575. https://doi.org/10.1016/j.jsg.2010.06.009

FERROUD, A.; RAFINI, S.; CHESNAUX, R. 2018. Using flow dimension sequences to interpret non-uniform aquifers with constant-rate pumping-tests: a review. Journal of Hydrology X, 100003. https://doi. org/10.1016/j.hydroa.2018.100003

GALANOS, I.; ROKOS, D. 2006. A statistical approach in investigating the hydrogeological significance of remotely sensed lineaments in the crystalline mountainous terrain of the island of Naxos, Greece. Hydrogeology Journal, 14(8): 1569-1581. https://doi. org/10.1007/s10040-006-0043-2

GRINGARTEN, A.C.; RAMEY Jr., H.J.. 1974. Unsteady-state pressure distributions created by a well with a single horizontal fracture, partial penetration, or restricted entry. Society of Petroleum Engineers Journal, 14(4): $413-$ 426. https://doi.org/10.2118/3819-PA

GRINGARTEN, A.C.; WITHERSPOON, P.A. 1972. A Method of Analyzing Pump Test Data from Fractured Aquifers. In: Int. Soc. Rock. Mech. / Int. Assoc. Eng. Geol., SYMPOSIUM OF PERCOLATION THROUGH FISSURED ROCK, Stuttgard, Proceeedings, vol. 3B, p. 1-9.

GUDMUNDSSON,A. 1992. Formation and growth of normal faults at the divergent plate boundary in Iceland. Terra Nova, 4(4): 464-471. https:// doi.org/10.1111/j.1365-3121.1992.tb00582.x

GUIHÉNEUF, N.; BOISSON, A.; BOUR, O.; DEWANDEL, B.; PERRIN, J.; DAUSSE, A.; MARÉCHAL, J.C. 2014. Groundwater flows in weathered crystalline rocks: Impact of piezometric variations and depth-dependent fracture connectivity. Journal of Hydrology, 511: 320-334. https://doi.org/10.1016/j. jhydrol.2014.01.061

HASUI, Y.; TOGNON, A.A.; SOARES, L.; CSORDAS, S.M. 1978. Geologia e tectônica da Serra do Japi. Boletim do Instituto de Geociências, 9: 17-24

HASUI, Y.; DANTAS, A.S.L.; CARNEIRO, C.D.R.; BISTRICHI, C.A. 1981. O embasamento pré-cambriano $e \quad o$ Eopaleozóico em São Paulo. In: Instituto de Pesquisas Tecnológicas do Estado de São Paulo, Mapa Geológico do Estado de São Paulo, escala 1:500.000. São Paulo: Publicação IPT, n. 1184, Monografias, 6: 12-45.

HOLBROOK, W.S.; MARCON, V.; BACON, A.R.; BRANTLEY, S.L.; CARR, B.J.; FLINCHUM, B.A.; RIEBE, C.S. 2019. Links between physical and chemical weathering inferred from a $65-\mathrm{m}$-deep borehole through Earth's critical zone. Scientific Reports, 9(1): 4495. https://doi.org/10.1038/s41598-01940819-9

IRRIGART - ENGENHARIA E CONSULTORIA EM RECURSOS HÍDRICOS E MEIO AMBIENTE. 2005. Caracterização das Bacias PCJ. Disponível em: http://www. agenciapcj.org.br/antigo/download/RS-0406_Capitulo-2.pdf. Acessado em 14 out. 2019.

JOURDE, H.; CORNATON, F.; PISTRE, S.; BIDAUX, P. 2002. Flow behavior in a dual fracture network. Journal of Hydrology, 266(1-2): 99-119. https://doi.org/10.1016/ S0022-1694(02)00120-8

LACHASSAGNE, P.; WYNS, R.; DEWANDEL, B. 2011. The fracture permeability of hard rock aquifers is due neither to tectonics, nor to unloading, but to weathering processes. Terra Nova, 23(3): 145-161. https://doi. org/10.1111/j.1365-3121.2011.00998.x

LE BORGNE, T.; BOUR, O.; DE DREUZY, J.R.; DAVY, P.; TOUCHARD, F. 2004. Equivalent mean flow models for fractured aquifers: Insights from a pumping tests scaling interpretation. Water Resources Research, 40(3): 1-12. https://doi. org/10.1029/2003WR002436

LEE, J.Y.; LEE, K.K. 2000. Use of hydrologic time series data for identification of recharge mechanism in a fractured bedrock aquifer system. Journal of Hydrology, 229(3-4): 
190-201. https://doi.org/10.1016/S00221694(00)00158-X

MABEE, S.B.; HARDCASTLE， K.C.; WISE, D.U. 1994. A method of collecting and analyzing lineaments for regionalscale fractured-bedrock aquifer studies. Groundwater, 32(6): 884-894. https://doi. org/10.1111/j.1745-6584.1994.tb00928.x

MADRUCCI, V. 2003. Sensoriamento remoto, aerogeofisica e geoprocessamento aplicados ao estudo de aquifero fraturado em terreno cristalino, leste do Estado de Sao Paulo. Revista Brasileira de Geociências, 33(suppl. 2): 43-52. https://doi.org/10.25249/0375$7536.200333 \mathrm{~S} 24352$

MANNING, C.E.; INGEBRITSEN, S.E. 1999. Permeability of the continental crust: Implications of geothermal data and metamorphic systems. Reviews of Geophysics, 37(1): 127-150. https://doi. org/10.1029/1998RG900002

MARÉCHAL， J.C.; DEWANDEL, B.; SUBRAHMANYAM, K. 2004. Use of hydraulic tests at different scales to characterize fracture network properties in the weathered-fractured layer of a hard rock aquifer. Water Resources Research, 40(11). https://doi.org/10.1029/2004WR003137

MOENCH, A.F. 1997. Flow to a well of finite diameter in a homogeneous, anisotropic water table aquifer. Water Resources Research, 33(6): 1397-1407. https://doi. org/10.1029/97WR00651

NETTLETON, W.D.; FLACH, K.W.; NELSON, R.E. 1970. Pedogenic weathering of tonalite in southern California. Geoderma, 4(4): 387-402. https://doi.org/10.1016/00167061(70)90055-8

NEVES, M.A.; MORALES, N. 2007. Well productivity controlling factors in crystalline terrains of southeastern Brazil. Hydrogeology Journal, 15(3): 471-482. https://doi. org/10.1007/s10040-006-0112-6

PAHL-WOSTL, C.; TÀBARA, D.; BOUWEN, R.; CRAPS, M.; DEWULF, A.; MOSTERT, E.; TAILLIEU, T. 2008. The importance of social learning and culture for sustainable water management. Ecological Economics, 64(3): 484-495. https://doi.org/10.1016/j. ecolecon.2007.08.007

PERSAUD, E.; LEVISON, J.; PEHME, P.; NOVAKOWSKI, K.; PARKER, B. 2018. Cross-hole fracture connectivity assessed using hydraulic responses during liner installations in crystalline bedrock boreholes. Journal of Hydrology, 556: 233-246. https:// doi.org/10.1016/j.jhydrol.2017.11.008

RAFINI, S.; LAROCQUE, M. 2012. Numerical modeling of the hydraulic signatures of horizontal and inclined faults. Hydrogeology Journal, 20(2): 337-350. https://doi. org/10.1007/s10040-011-0812-4

REIMUS, P.; POHLL, G.; MIHEVC, T.; CHAPMAN, J.; HAGA, M.; LYLES, B.; KOSINSKI, S.; NISWONGER, R.; SANDERS, P. 2003. Testing and parameterizing a conceptual model for solute transport in a fractured granite using multiple tracers in a forced-gradient test. Water Resources Research, 39(12): 1-14. https:// doi.org/10.1029/2002WR001597

RENARD, P.; GLENZ, D.; MEJIAS, M. 2009. Understanding diagnostic plots for well-test interpretation. Hydrogeology Journal, 17(3): 589-600. https://doi.org/10.1007/s10040008-0392-0

ROQUES, C.; BOUR, O.; AQUILINA, L.; DEWANDEL, B. 2016. High-yielding aquifers in crystalline basement: insights about the role of fault zones, exemplified by Armorican Massif, France. Hydrogeology Journal, 24(8): 2157-2170. https://doi. org/10.1007/s10040-016-1451-6

SHIKLOMANOV, I.A. 2000. Appraisal and assessment of world water resources. Water International, 25(1): 11-32. https://doi. org/10.1080/02508060008686794

STOBER, I.; BUCHER, K. 2007. Hydraulic properties of the crystalline basement. Hydrogeology Journal, 15(2): 213-224. https://doi.org/10.1007/s10040-007-0214-9

TAYLOR, R.; HOWARD, K. 2000. A tectonogeomorphic model of the hydrogeology of deeply weathered crystalline rock: evidence 
from Uganda. Hydrogeology Journal, 8(3): 279-294. https://doi.org/10.1007/ s100400000069

THEIS, C.V. 1935. The relation between the lowering of the piezometric surface and the rate and duration of discharge of a well using ground-water storage. Eos, Transactions American Geophysical Union, 16(2): 519-524. https://doi.org/10.1029/ TR016i002p00519

VOUILLAMOZ, J.M.; DESCLOITRES, M.; TOE, G.; LEGCHENKO, A. 2005. Characterization of crystalline basement aquifers with MRS: comparison with boreholes and pumping tests data in Burkina Faso. Near Surface Geophysics, 3(3): 205-213. https://doi. org/10.3997/1873-0604.2005015

WRIGHT, E. P. 1992. The hydrogeology of crystalline basement aquifers in Africa. Geological Society, London, Special Publications, 66(1): 1-27. https://doi. org/10.1144/GSL.SP.1992.066.01.01

ZHAO, J. 1998. Rock mass hydraulic conductivity of the Bukit Timah granite, Singapore. Engineering Geology, 50(1-2): 211-216. https://doi.org/10.1016/S0013-7952(98)00021-0

\section{Endereço dos autores:}

Fábio Cruz - FRC Serviços Geológicos e Ambientais Ltda, Rua Congo, 313, Torre 2, Apt. 106, Bairro Jardim Bonfiglioli, 313, Jardim Bonfiglioli, CEP 13207-340, Jundiaí, SP. E-mails: fabiocruzbr@yahoo. com.br

Elias Hideo Teramoto - Laboratório de Estudos de Bacias - UNESP e Centro de Estudos Ambientais UNESP, Campus de Rio Claro, Av. 24-A, 1515, Bairro Bela Vista, CEP 13506-900, Rio Claro, SP, Brasil. E-mail: elias.hideo-teramoto@unesp.br

Juan Navarro - Laboratório de Estudos de Bacias - UNESP, Campus de Rio Claro, Av. 24-A, 1515, Bairro Bela Vista, CEP 13506-900, Rio Claro, SP, Brasil.E-mail: j.navarro@unesp.br

Hung Kiang Chang - Laboratório de Estudos de Bacias - UNESP, Centro de Estudos Ambientais - UNESP e Departamento de Geologia Aplicada, Instituto de Geociências e Ciências Exatas - UNESP, Campus de Rio Claro, Av. 24-A, 1515, Bairro Bela Vista, CEP: 13506-900, Rio Claro, SP, Brasil. E-mail: chang. hung-kiang@unesp.br

Artigo submetido em 3 de novembro de 2019, aceito em 20 de dezembro de 2019. 